PUBLICATION VI

\title{
Erbium-doped waveguides fabricated with atomic layer deposition method
}

In: IEEE Photonics Technology Letters 2004.

Vol. 16, No. 1, pp. 194-196.

Reprinted with permission from the publisher.

(C) 2004 IEEE 


\title{
Erbium-Doped Waveguides Fabricated With Atomic Layer Deposition Method
}

\author{
Kimmo Solehmainen, Markku Kapulainen, Päivi Heimala, and Kirsi Polamo
}

\begin{abstract}
Atomic layer deposition was used in preparing erbium (Er)-doped waveguides. Ridge-type Er-doped $\mathrm{Al}_{2} \mathrm{O}_{3}$ waveguides were patterned on silica-coated silicon wafers using photolithography and wet etching. Optical absorption, emission, fluorescence lifetime, and signal enhancement measurements were performed. Polarization dependence of the absorption spectrum and birefringence of the waveguide were measured. The material showed strong absorption and wide emission spectrum around $1530 \mathrm{~nm}$ with full-width at half-maximum of $52 \mathrm{~nm}$. Signal enhancement of $6 \mathrm{~dB}$ was measured for a 3.9-cm-long waveguide.
\end{abstract}

Index Terms-Aluminum oxide, atomic layer deposition (ALD), erbium (Er), optical amplification, optical waveguides.

\section{INTRODUCTION}

$\mathbf{E}$ RBIUM (Er)-doped optical waveguides offer a means for optical amplification or compensation for the losses present in passive integrated optical circuits. In addition to the flexible integration, planar technology can offer cost efficiency through mass production when compared to the fiber-optical amplifiers. Several different techniques have been used to produce Er-doped waveguides [1]-[5].

The cost-efficiency of the planar optical amplifiers is only achieved if large-area thin films can be grown with a sufficient uniformity and a precise thickness control. Atomic layer deposition (ALD), also known as atomic layer epitaxy, excellently addresses these issues. In ALD, the thin film is grown in a sequential manner. The deposition is based on surface reactions of the precursor gases, which are introduced in the deposition chamber one at a time. The process consists of a selected amount of reaction cycles and it allows basically a nanometer accuracy in the material growth control. Generally, the film is deposited conformally regardless of the surface shape. Temperature is kept relatively low during the growth, typically below $400{ }^{\circ} \mathrm{C}$. Low processing temperature ensures that the thermally induced stress in the deposited film stack remains reasonably low.

A wide range of materials has been grown with ALD [6]. It is suitable for various applications and today it has also been adopted by large semiconductor manufacturers. In optical applications, ALD has been used since 1980 for depositing large-area electroluminescence-based thin-film displays [7]. It has also been used for preparation of dielectric multilayer structures for some basic optical components such as antire-

Manuscript received June 27, 2003; revised September 1, 2003.

K. Solehmainen, M. Kapulainen, and P. Heimala are with VTT Information Technology, Microelectronics, FIN-02044 VTT Espoo, Finland (e-mail: kimmo.solehmainen@vtt.fi).

K. Polamo is with Planar Systems, Inc., FIN-02201 Espoo, Finland (e-mail: kirsi_polamo@planar.com).

Digital Object Identifier 10.1109/LPT.2003.820484 flection and high-reflection coatings, neutral beam splitters, and Fabry-Pérot filters [8]. Nonetheless, it has not been a common technique for fabricating integrated optical components. In this field, the excellent uniformity of ALD can offer a well-controlled device operation, e.g., in the phase sensitive devices. In addition to this, ALD offers a way to fabricate antireflective or reflective waveguide end coatings as well as to connect different waveguide sections with low loss, both these directly on a wafer level. This is possible due to the conformal growth of ALD. Since ALD has been used for over two decades for fabrication of electroluminescence-based displays, it is a natural step to investigate rare-earth doped ALD films also for other applications. In this letter, results on the ALD-grown Er-doped $\mathrm{Al}_{2} \mathrm{O}_{3}$ waveguides are presented. Aluminum oxide has been used as a host material for Er, because the ALD process for $\mathrm{Al}_{2} \mathrm{O}_{3}$ is well known and the Er solubility in $\mathrm{Al}_{2} \mathrm{O}_{3}$ is reasonable high.

\section{EXPERIMENT}

Ridge-type waveguides were formed on 2 - $\mu \mathrm{m}$-thick ALD-grown $\mathrm{Al}_{2} \mathrm{O}_{3}$ films on silicon substrate. A 5- $\mu$ m-thick plasma-enhanced chemical vapor deposition (PECVD)-grown silica film was used as an under cladding. Er-doping was done by adding Er cycles between the $\mathrm{Al}_{2} \mathrm{O}_{3}$ cycles in the ALD process. The Er concentration was measured with X-ray fluorescence resulting in an average Er concentration of $2.3 \mathrm{wt} \%$. The measurement method did not take into account the layered doping structure. The measured doping level corresponds to the Er ion concentration of $3.2 \times 10^{20} \mathrm{~cm}^{-3}$ (assuming density of $4.0 \mathrm{~g} / \mathrm{cm}^{3}$ ).

For the ridge waveguide formation, a thin molybdenum (MO) film was sputtered as a hard mask for the $\mathrm{Al}_{2} \mathrm{O}_{3}$ etching. The Mo film was patterned with standard photolithography and wet etching in a commercial aluminum etchant (PS 70/10). The $\mathrm{Al}_{2} \mathrm{O}_{3}$ wet etching in $50 \%$ phosphoric acid at $75^{\circ} \mathrm{C}$ for $7.5 \mathrm{~min}$ was followed in order to define the waveguide structure. This resulted in a ridge height of $390 \mathrm{~nm}$ as measured with a profilometer. After the resist removal the samples were immersed in $\mathrm{NH}_{4} \mathrm{OH}-\mathrm{H}_{2} \mathrm{O}_{2}$-deionized water solution to remove the Mo mask. No upper cladding was used on top of the waveguides. Finally, the waveguides were cleaved in order to perform the optical characterization.

The waveguide structure was selected to fulfill a single-mode operation, but it was not optimized for the low-loss fiber coupling. The realized waveguide structure was examined with an optical microscope and the measured dimensions were used for the mode-field simulations. The simulations were done with the TempSelene program, which uses the finite difference method. 


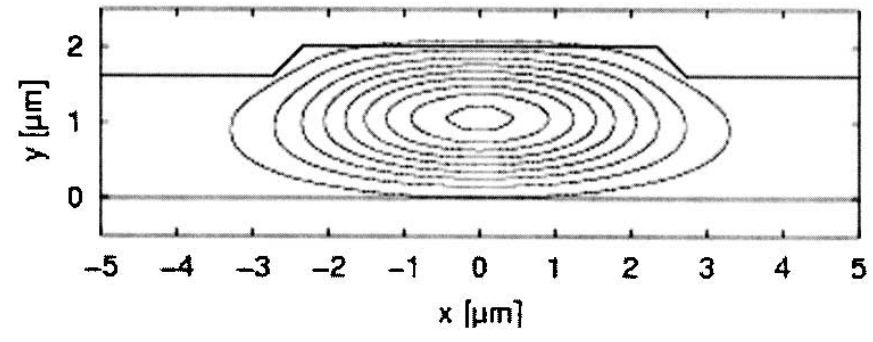

Fig. 1. Simulated cross-sectional power distribution of the fundamental TM mode for a nominally $6.0-\mu \mathrm{m}$-wide ridge-type waveguide. The ridge height is $390 \mathrm{~nm}$. The contours are for normalized intensities $0.1,0.2, \ldots$, and 0.9 .

Resulting cross-sectional power distribution of the fundamental transverse magnetic (TM) mode for a nominally $6.0-\mu \mathrm{m}$-wide waveguide is shown in Fig. 1.

From the cleaved waveguides, optical transmission, emission, fluorescence lifetime, and signal gain characteristics were measured. The measured straight waveguide sample was $3.9 \mathrm{~cm}$ long. The width of the measured waveguide was $6.0 \mu \mathrm{m} \mathrm{ex}-$ cept in the emission spectrum and fluorescence lifetime measurements, where the waveguide width was $2.8 \mu \mathrm{m}$.

The polarization dependence of the transmission spectrum was measured, because it was observed that the waveguides were birefringent. In the measurement setup, the input light was butt-coupled into the waveguide with a single-mode polarization-maintaining fiber. The setup enabled the transmission measurement of both transverse electric (TE) and TM polarizations independently. The transmitted light was coupled into a multimode fiber and guided to an optical spectrum analyzer. The birefringence, i.e., the difference between the effective refractive indexes of TE and TM modes, of the waveguide was measured using the fixed analyzer method [9].

In the emission spectrum measurement, the $\mathrm{Er}$ ions in the waveguide were excited using a 980-nm pump source. Light from a 980-nm diode laser was coupled to the waveguide with a standard single-mode fiber. Backward-directed emission light was gathered back to the same fiber. A 1550/980-nm wavelength-division multiplexer (WDM) was used to filter the pump light from the emitted light before feeding the emitted light to an optical spectrum analyzer.

The fluorescence lifetime was measured with the same setup as the emission spectrum. The only differences were that the backward-directed emission light was fed to an InGaAs photodetector and the pump laser was modulated with a pulse generator. The electrical signal of the photodetector was measured with a digitizing oscilloscope. The fluorescence lifetime was defined as a time during which the emitted intensity from the waveguide dropped to $1 / \mathrm{e}$ of the initial intensity after the pump was switched off.

In the gain spectrum measurement, the waveguide was pumped with a 980-nm laser and the signal was provided by a tunable narrow-band laser. The signal and the pump light were combined with a 1550/980-nm WDM to a single-mode fiber, which was coupled to the waveguide input. Light from the waveguide output was coupled to a multimode fiber and measured with an optical spectrum analyzer.

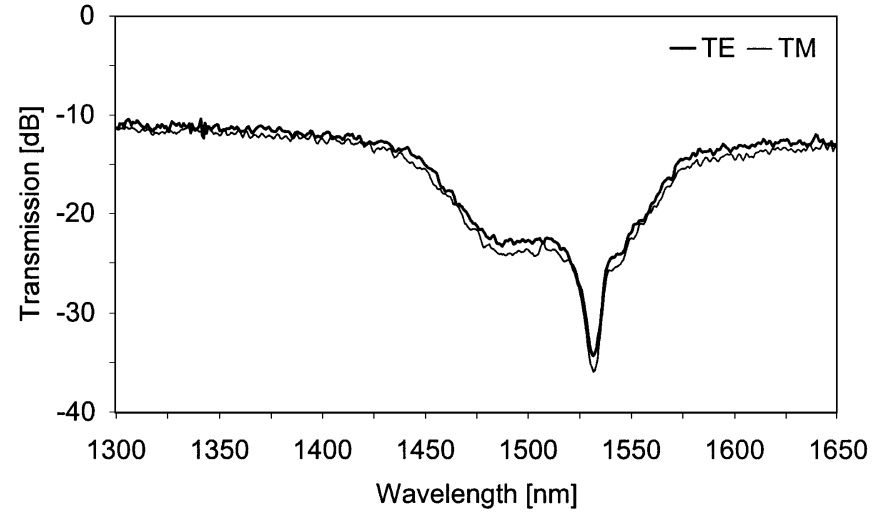

Fig. 2. Transmission spectra of a 3.9-cm-long Er-doped waveguide for TE and TM polarizations.

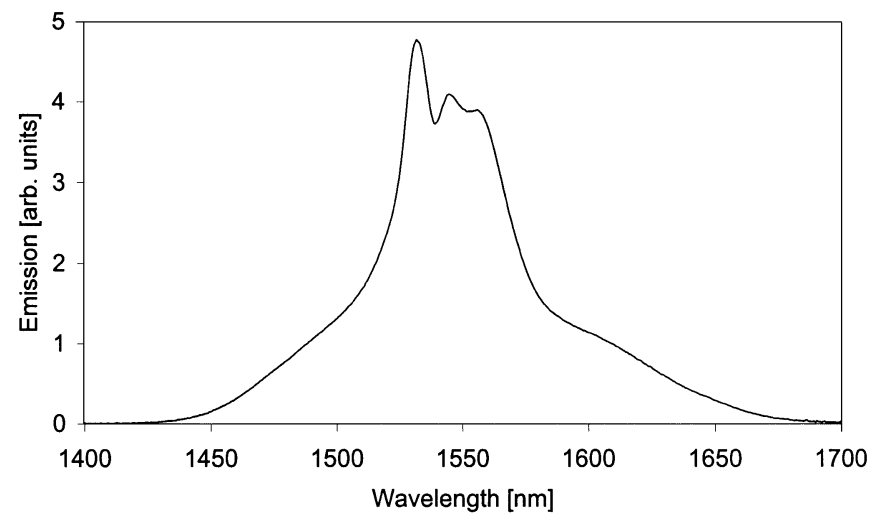

Fig. 3. Emission spectrum of an Er-doped waveguide. The FWHM of the spectrum is $52 \mathrm{~nm}$.

\section{RESULTS AND DISCUSSION}

Measured transmission spectra for both TE and TM polarizations of an Er-doped waveguide are shown in Fig. 2. The spectra show clearly the absorption due to the Er ions, the maximum being $6.1 \mathrm{~dB} / \mathrm{cm}$ for $\mathrm{TE}$ and $6.4 \mathrm{~dB} / \mathrm{cm}$ for $\mathrm{TM}$ at $1530 \mathrm{~nm}$. The maximum absorption measured for nonpolarized light was $6.2 \mathrm{~dB} / \mathrm{cm}$. The birefringence of this waveguide around $1550 \mathrm{~nm}$ was measured as 0.004 , which coincided with the simulated birefringence value for a corresponding waveguide structure. The birefringence can be lowered by modifying the waveguide structure. The background losses can be estimated from the transmission values around $1300 \mathrm{~nm}$, where no Er-induced absorption occurs. In Fig. 2, the background losses are about $11 \mathrm{~dB}$. According to the simulations, this figure contains $4.5 \mathrm{~dB}$ of fiber coupling losses. The rest consists of fiber misalignment and propagation losses. Since the wet etching does not increase the surface roughness of the waveguides considerably, the high propagation losses are expected to be caused by scattering in the material.

Fig. 3 shows the measured emission spectrum of an Er-doped waveguide. The pump power in the input fiber was $100 \mathrm{~mW}$, of which about $35 \mathrm{~mW}$ reached the waveguide. The highest peak of the emission spectrum is around $1530 \mathrm{~nm}$, followed by a broad emission shoulder from 1540 to $1560 \mathrm{~nm}$. The full-width at half-maximum (FWHM) of the emission spectrum is $52 \mathrm{~nm}$. 


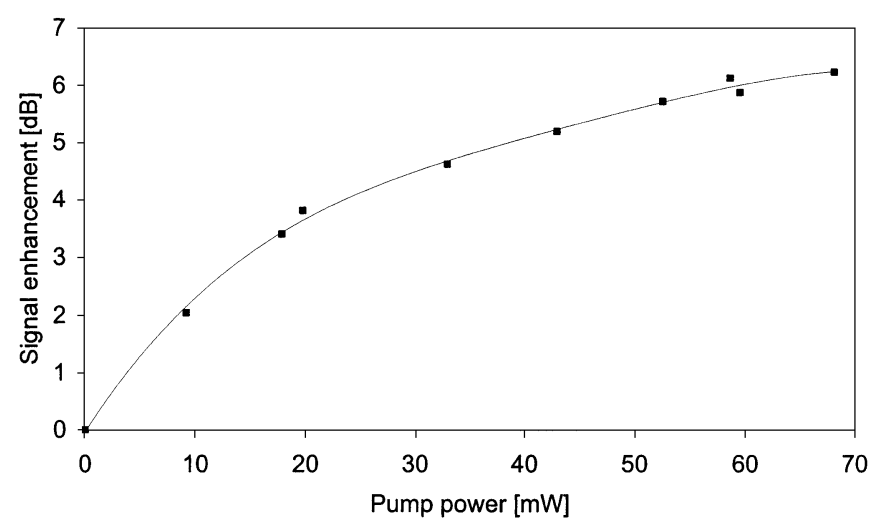

Fig. 4. Signal enhancement of an Er-doped waveguide as a function of the pump power at $1550-\mathrm{nm}$ signal wavelength. The pump wavelength was $980 \mathrm{~nm}$.

The broad emission spectrum is typical for Er-doped $\mathrm{Al}_{2} \mathrm{O}_{3}$ material, being broader than for Er-doped silica [10].

In the gain measurement, the absorption of the signal light competed with the stimulated emission and net optical gain was not reached. This indicates that the inversion of the Er ions was not sufficient. However, the signal at the output was clearly amplified when the pump was switched on. The ratio of the output signal with and without the pump power is commonly referred to as the signal enhancement. The signal enhancement at $1550 \mathrm{~nm}$ as a function of pump power is shown in Fig. 4. The pump power given in the figure indicates the optical power in the input fiber. The actual power in the waveguide is estimated to be $65 \%$ lower. As can be seen from the figure, the signal enhancement saturates to a level of about $6 \mathrm{~dB}$ when the pump power is increased.

The measured fluorescence lifetime of $0.9 \mathrm{~ms}$ verified the poor inversion of the Er ions. The short lifetime is most probably due to the inhomogeneous doping profile generated by the fabrication method. The pure Er layers between the $\mathrm{Al}_{2} \mathrm{O}_{3}$ layers with a locally high volume density of the Er ions cause the doping profile to be inhomogeneous. This is expected to increase the cooperative upconversion of the Er ions, especially if ions in the Er layer are clustered [11]. The spreading of the doping profile would most likely increase the fluorescence lifetime and enable net optical gain in these waveguides. This is hard to realize with annealing because the diffusivity of the $\mathrm{Er}$ ions in $\mathrm{Al}_{2} \mathrm{O}_{3}$ is low. However, the spreading of Er ions within a single doping layer by codoping (e.g., with ytterbium) or by using other elements to adjust the Er ion density within a single layer in the ALD process should be fully examined to improve the lifetime characteristics.

\section{CONCLUSION}

In this letter, the fabrication of Er-doped $\mathrm{Al}_{2} \mathrm{O}_{3}$ waveguides using ALD was demonstrated. The ridge-type waveguides were processed on a silicon substrate with a PECVD silica buffer layer. The measured waveguides showed a broad emission spectrum with FWHM over $50 \mathrm{~nm}$ and strong Er-induced absorption, the maximum being $6.2 \mathrm{~dB} / \mathrm{cm}$ for nonpolarized light. The birefringence of the measured waveguide was 0.004 . The birefringence influenced only moderately on the polarization dependence of the optical transmission. Signal enhancement saturated to about $6 \mathrm{~dB}$ for a 3.9-cm-long waveguide. To reach a net optical gain, the doping process needs further development to realize a more uniform doping profile throughout the Er-doped active layer.

\section{ACKNOWLEDGMENT}

The authors would like to thank Dr. R. Törnqvist for the technical support during this work, M. Partanen for processing of the waveguides, and M. Harjanne and T. Aalto for the simulation work.

\section{REFERENCES}

[1] Y. C. Yan, A. J. Faber, H. de Waal, P. G. Kik, and A. Polman, "Erbium-doped phosphate glass waveguide on silicon with $4.1 \mathrm{~dB} / \mathrm{cm}$ gain at $1.535 \mu \mathrm{m}, "$ Appl. Phys. Lett., vol. 71, pp. 2922-2924, 1997.

[2] D. Barbier, P. Bruno, C. Cassagnettes, M. Trouillon, R. L. Hyde, A. Kevorkian, and J. M. P. Delavaux, "Net gain of $27 \mathrm{~dB}$ with a 8.6-cm-long Er/Yb-doped glass-planar-amplifier," in Proc. OFC'98, 1998 , pp. 45-46.

[3] K. Hattori, T. Kitagawa, M. Oguma, Y. Ohmori, and M. Horiguchi, "Erbium-doped silica-based waveguide amplifier integrated with a 980/1530 nm WDM coupler," Electron. Lett., vol. 30, pp. 856-857, 1994.

[4] X. Orignac, D. Barbier, X. M. Du, R. M. Almeida, O. McCarthy, and E. Yeatman, "Sol-gel silica/titania-on-silicon Er/Yb-doped waveguides for optical amplification at $1.5 \mu \mathrm{m}$," Opt. Mater., vol. 12, pp. 1-18, 1999.

[5] K. Shuto, K. Hattori, T. Kitagawa, Y. Ohmori, and M. Horiguchi, "Erbium-doped phosphosilicate glass waveguide amplifier fabricated by PECVD," Electron. Lett., vol. 29, pp. 139-141, 1993.

[6] L. Niinistö, "Advanced thin films for electronics and optoelectronics by atomic layer epitaxy," in Proc. Int. Semiconductor Conf. CAS 2000, vol. 1, 2000, pp. 33-42.

[7] T. Suntola, J. Antson, A. Pakkala, and S. Lindfors, "Atomic layer epitaxy for producing EL-thin films," in Proc. SID 80 Dig., 1980, pp. 108-109.

[8] D. Riihelä, M. Ritala, R. Matero, and M. Leskelä, "Introducing atomic layer epitaxy for the deposition of optical thin films," Thin Solid Films, vol. 289, pp. 250-255, 1996.

[9] Y. Namihira and J. Maeda, "Comparison of various polarisation mode dispersion measurement methods in optical fibers," Electron. Lett., vol. 28, pp. 334-335, 1992.

[10] T. Kitagawa, K. Hattori, M. Shimizu, Y. Ohmori, and M. Kobayashi, "Guided-wave laser based on erbium-doped silica planar lightwave circuit," Electron. Lett., vol. 27, no. 1991, pp. 334-335.

[11] G. N. van den Hoven, E. Snoeks, A. Polman, C. van Dam, J. W. M. van Uffelen, and M. K. Smit, "Upconversion in Er-implanted $\mathrm{Al}_{2} \mathrm{O}_{3}$ waveguides," J. Appl. Phys., vol. 79, pp. 1258-1266, 1996. 